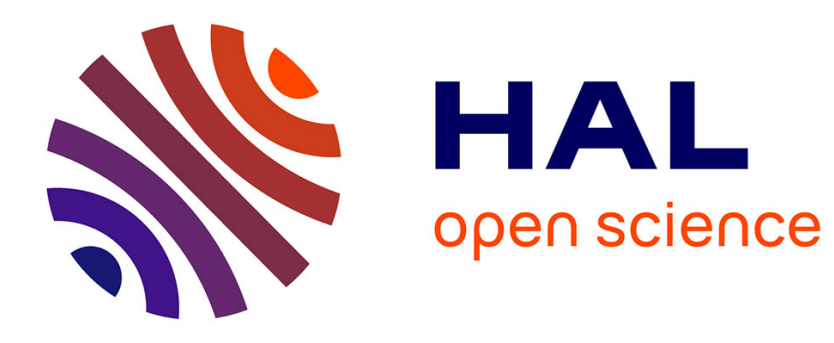

\title{
Optimal placement of base stations in wireless indoor telecommunication
}

Thom Frühwirth, Pascal Brisset

\section{To cite this version:}

Thom Frühwirth, Pascal Brisset. Optimal placement of base stations in wireless indoor telecommunication. CP 98, 4th International Conference on Principles and Practice of Constraint Programming, Oct 1998, Pisa, Italy. pp 476-480, 10.1007/3-540-49481-2_47 . hal-01021683

\section{HAL Id: hal-01021683 \\ https://hal-enac.archives-ouvertes.fr/hal-01021683}

Submitted on 21 Nov 2014

HAL is a multi-disciplinary open access archive for the deposit and dissemination of scientific research documents, whether they are published or not. The documents may come from teaching and research institutions in France or abroad, or from public or private research centers.
L'archive ouverte pluridisciplinaire HAL, est destinée au dépôt et à la diffusion de documents scientifiques de niveau recherche, publiés ou non, émanant des établissements d'enseignement et de recherche français ou étrangers, des laboratoires publics ou privés. 


\title{
Optimal Placement of Base Stations in Wireless Indoor Telecommunication ${ }^{\star}$
}

\author{
Thom Frühwirth ${ }^{1}$ Pascal Brisset ${ }^{2}$ \\ 1 Ludwig-Maximilians-Universität München (LMU) \\ Oettingenstrasse 67, D-80538 Munich, Germany \\ fruehwir@informatik.uni-muenchen.de \\ http://www.informatik.uni-muenchen.de/ fruehwir/ \\ 2 Ecole Nationale de l'Aviation Civile (ENAC) \\ 7 Av. Edouard Belin, BP 4005, F-31055 Toulouse Cedex, France \\ Pascal.Brisset@recherche.enac.fr
}

\begin{abstract}
Planning of local wireless communication networks is about installing base stations (small radio transmitters) to provide wireless devices with strong enough signals. POPULAR is an advanced industrial prototype that allows to compute the minimal number of base stations and their location given a blue-print of the installation site and information about the materials used for walls and ceilings. It does so by simulating the propagation of radio-waves using ray tracing and by subsequent optimization of the number of base stations needed to cover the whole building. Taking advantage of state-of-the-art techniques for programmable application-oriented constraint solving, POPULAR is among the first practical tools that can optimally plan wireless communication networks.
\end{abstract}

\section{Introduction}

Mobile communication has become literally ubiquitous these days. According to a press release of British Telecommunications Plc and MCI Communications Corp of September 1996, there are 60 Mill. mobile phones worldwide (compare this figure to the world's 50 Mill. fax numbers and 40 Mill. email addresses). More and more, mobile communications also comes to company sites by means of local, indoor wireless communication networks. No cabling is required and the employees can be reached at any time at any place.

Current systems are cellular in that a base station (sender, transmitter) controls the links to the tranceivers. A (radio) cell is the space that is covered by a single base station. For buildings, multi-cellular systems are required, because walls and floors absorb part of the radio signal.

Today, the number and positioning of base stations is estimated by an experienced sales person. Computer-aided planning promises to ease some of the

\footnotetext{
* Work was done while the authors were at ECRC, Munich, Germany
} 
difficulties encountered. An advanced prototype, POPULAR (Planning of Picocellular Radio) $[3,4]$, was developed in collaboration with industry and research institutions in Germany: The Siemens Research and Development Department (ZFE), the Siemens Personal Networks Department (PN), the European Computer-Industry Research Center (ECRC) and the Institute of Communication Networks at the Aachen University of Technology.

Given a blue-print of the building and information about the materials used for walls and ceilings, POPULAR computes the minimal number of base stations and their location by simulating the propagation of radio-waves using ray tracing and subsequent optimization of the number of base stations needed to cover the whole building.

The authors implemented a first protoype in a few months in the constraint logic programming language $\mathrm{ECL}^{i} \mathrm{PS}^{e}[6]$ while at ECRC in 1995. The language includes a library for Constraint Handling Rules (CHR) [5], which are a highlevel language extension to implement arbitrary constraint systems. The CHR library was essential for a rapid, flexible and efficient implementation of the constraints that appear in this optimization problem. Based on this prototype, J.-R. Molwitz, a student from the University of Aachen, implemented POPULAR within one man-year while at Siemens.

\section{Simulation of Radio Cells by Ray-Tracing}

Radio wave propagation suffers mainly from the following effects:

- attenuation (weakening) of the signal due to distance,

- shadowing (absorption) through obstacles,

- multipath propagation due to reflection and diffraction.

The COST ${ }^{3}$ Subgroup 'Propagation Models' proposed the so-called path loss model [1] to describe these effects. The model is based on the power balance of wireless transmission. It combines a distance dependent term with correction factors for extra path loss due to floors and walls of the building in the propagation path. To take reflection and multipath effects into account, a fading reserve (fade margin) is introduced. We also extended the model to take the directional effect of an antenna into account, since antennas do not beam with the same energy in every direction.

In the simulation phase, the characteristics of the building are computed using of test points. Each test point represents a possible receiver position. The test points are placed on a 3 -dimensional grid inside the volume that should be covered. For each test point the space where a base station can be put to cover the test point, the "radio cell", is calculated. If the test grid is sufficiently small (several per squaremeter), we can expect that if two neighbouring test points are covered, the space inbetween - hence the whole building - can also be covered.

Ray tracing simulates the propagation of radio waves through the walls and ceilings of the building. To get to the point of minimal sensitivity (i.e. maximal

\footnotetext{
${ }^{3}$ European Cooperation in the field Of Scientific and Technical research
} 
permissible path loss), each path must be followed through the whole building. The values of antenna attenuation in the direction of the path, the path loss due to the distance and the insertion losses due to intersections of the path with walls and floors are added up to the maximal permissible path loss. The resulting end points are used to describe the hull of the radio cell. Note that the radio cell will usually be a rather odd-shaped object, since the received power may exhibit discontinuities because of tiny changes in the location - such as a move around the corner.

\section{Constraint-Based Optimization}

For each of the resulting radio cells a constraint is set up that there must be a location of a base station (geometrically speaking, a point) somewhere in that space. Then, we try to find locations that are in as many cells at the same time as possible. This means that a base station at one of these locations will cover several test points at once. Thus the possible locations are constrained to be in the intersections of the cells covered. In this way, a first solution is computed. Next, to minimize the number of base stations, we use a branch-and-bound method. It consists in repeatedly searching for a solution with a smaller number of base stations until the minimal number is found. The constraint solver was implemented using Constraint Handling Rules (CHR) [5], which are essentially multi-headed guarded rules that rewrite constraints into simpler ones until they are solved.

In a first attempt restricted to two dimensions, we approximated a cell by a single rectangle. The 2-D coordinates are of the form $\mathrm{X \# Y}$, rectangles are orthogonal to the coordinate system and are represented by a pair, composed of their left lower and right upper corner coordinates. For each cell, simply a constraint inside(Sender, Rectangle) is imposed, where Sender refers to a point that must be inside the Rectangle.

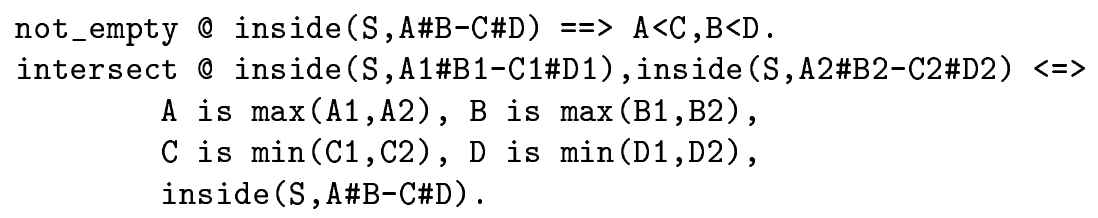

The first rule (named not_empty) says that the constraint inside ( $\mathrm{S}, \mathrm{A \# B-C \# D)}$ is only valid if also the condition $A \angle C, B<D$ is fulfilled, so that the rectangle has a non-empty area. The intersect rule says that if a base station location $\mathrm{S}$ is constrained by two inside constraints to be in two rectangles at once, we can replace these two constraints by a single inside constraint whose rectangle is computed as the intersection of the two initial rectangles.

To compute a solution, after we have set up all the inside constraints, we try to equate as many base stations as possible. Equating base stations causes the intersect rule to fire with the constraints associated with the base stations. As a result of this labeling procedure, a base stations location will be constrained more and more and thus the intersect rule will be applied again and again 
until the rectangle becomes very small and finally empty. Then the not_empty rule applies, causes failure and so initiates chronological backtracking that will lead to another choice.

It took just 10 minutes to extend this solver so that it works with union of rectangles, that can describe the cell more accurately - actually to any desired degree of precision. The union corresponds to a disjunctive constraint of the form inside $(S, R 1)$ or inside (S,R2) or ... or inside (S,Rn) which is more compactly implemented as inside ( $\mathrm{S},[\mathrm{R} 1, \mathrm{R} 2, \ldots, \mathrm{Rn}])$. The subsequent lifting to 3 dimensions just amounted to adding a third coordinate and code analogous to the one for the other dimensions.

\section{Evaluation}

Taking advantage of state-of-the-art techniques for programmable applicationoriented constraint solving, POPULAR was among the first practical tools that could optimally plan wireless communication networks. While we worked on POPULAR, without knowing from each other, the WiSE tool [2] was developed with exactly the same functionality. WiSE is written in about 7500 lines of $\mathrm{C}++$. For optimization WiSE uses an adaptation of the Nelder-Mead direct search method that optimizes the percentage of the building covered. WiSE has been patented and is in commercial use by Lucent Technologies since 1997 to plan their DEFINITY Wireless Business System - PWT. Another approach [7] uses the Nelder-Mead method for continuous space and Hopfield Neural Networks for a modelling in discrete space. The authors shortly mention a tool called IWNDT written in $\mathrm{C}$.

For a typical office building, an optimal placement is found by POPULAR within a few minutes. This is impressive since everything (including ray tracing and a graphical user interface) was implemented in a CLP language. The CLP code is just about 4000 lines with more than half of it for graphics and user interface. The overall quality of the placements produced is comparable to that of a human expert. The precision is influenced by the underlying path loss model with its the fading reserve, the number of rays used in the simulation and the approximation of radio cells by unions of rectangles.

The result of covering a medieval monastery is shown in Figure 1, where four base stations are needed. If more than one base station covers a region, it is attributed to the base station that provides the strongest signal.

\section{References}

[1] COST 231 'Propagation Models' Subgroup, Building Penetration Losses, Report COST 231 TD (90) 116, Darmstadt, Germany, December 1990.

[2] S. J. Fortune, D. M. Gay, B. W. Kernighan et al., WiSE Design of Indoor Wireless Systems, IEEE Computational Science and Engineering, Vol. 2, No. 1, pp. 58-68, Spring, 1995. 


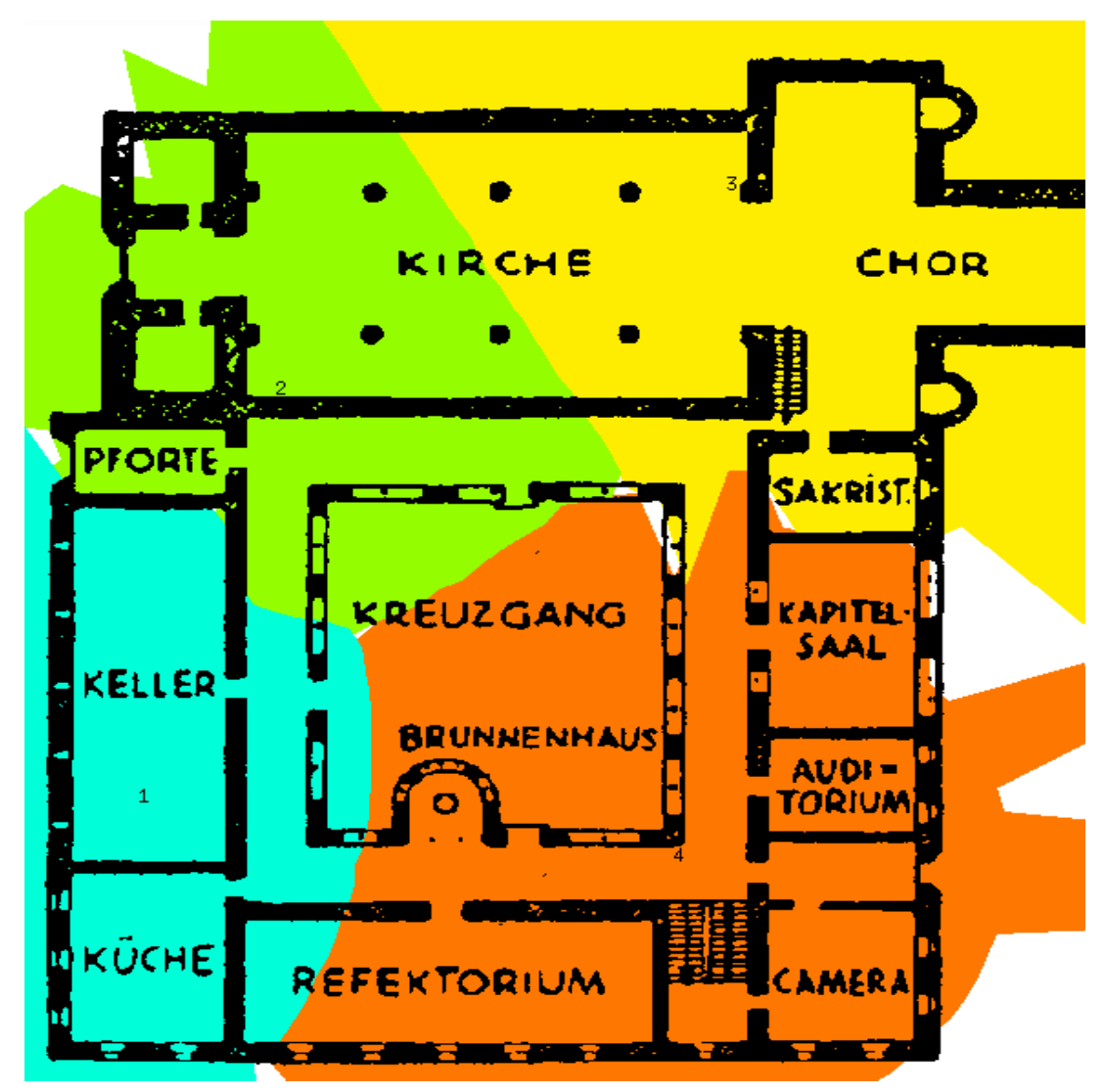

Fig. 1. Covering a medieval monastery

[3] T. Frühwirth, J.-R. Molwitz and P. Brisset, Planning Cordless Business Communication Systems, IEEE Expert Magazine, Special Track on Intelligent Telecommunications, February 1996.

[4] T. Frühwirth and P. Brisset, Optimal Planning of Digital Cordless Telecommunication Systems, Third International Conference on The Practical Application of Constraint Technology (PACT97), London, U.K., April 1997.

[5] T. Frühwirth, Theory and Practice of Constraint Handling Rules, Special issue on constraint logic programming (K. Marriott and P. J. Stuckey, Eds.), Journal of Logic Programming, Vol 37(1-3), pp 95-138, October 1998.

[6] M. Wallace, St. Novello and J. Schimpf, ECLiPSe: A Platform for Constraint Logic Programming, Technical Report, IC-Parc, Imperial College, London, August 1997.

[7] D. Stamatelos and A. Ephremides, Spectral Efficiency and Optimal Base Placement for Indoor Wireless Networks, IEEE Journal on Selected Areas in Communications, Vol. 14, No.4, May 1996.

This article was processed using the IATEX macro package with LLNCS style 